\title{
Can Non-alcoholic fatty liver disease (NAFLD) as a marker for microalbuminuria in prediabetes group?
}

\author{
Anjaneya Prasad V 1 Sirisha Y 2 Pradeep Babu K V 3 \\ ${ }^{1 .}$ Associate Professor, Department of Internal Medicine, Dr.PSIMS\&RF, Chinoutpally, Gannavaram Mandal, \\ Krishna District, Andhra Pradesh. INDIA. 521286 \\ ${ }^{2 .}$ Resident, Department of Internal Medicine, Dr.PSIMS\&RF, Chinoutpally, Gannavaram Mandal, Krishna \\ District, Andhra Pradesh. INDIA. 521286 \\ ${ }^{3 .}$ Resident, Department of Internal Medicine, Dr.PSIMS\&RF, Chinoutpally, Gannavaram Mandal, Krishna \\ District, Andhra Pradesh. INDIA. 521286
}

\begin{abstract}
Objective: Non - alcoholic fatty liver disease (NAFLD) is an imageological entity without elevation of liver enzymes. The purpose of this case - control study was to corelate microalbuminuria to Prediabetes with and without NAFLD.

Method: This study was done at the Department of Medicine,DR. PSIMS \& RF Chinaoutpally, A.P. India between Jan 2012 to Dec 2012. 200 patients (100 cases, 100 controls) above 18 years of age were included in this study. The microalbuminuria reference range was $30-300 \mathrm{mg} /$ day.

Results: Microalbuminuria levels were found to be significantly higher in Prediabetes patients with non alcoholic fatty liver disease than prediabetes alone.
\end{abstract}

Key words: NAFLD, Prediabetes, Microalbuminuria

\section{INTRODUCTION:}

Non - alcoholic fatty liver disease (NAFLD) is a rapidly growing health problem in India along with other diseases like diabetes, hypertension, dyslipidemia and obesity $(1,2,3)$. The association of NAFLD with the features of the metabolic syndrome has raised an interest in its role in the development and progression of chronic kidney disease (CKD) and coronary artery disease (CAD). Many studies suggested that NAFLD, CAD and CKD share common risk factors and pathogenic mechanisms and that NAFLD, in particular, is associated with an increased prevalence and incidence of these diseases $(4,5)$. This association appears to be independent of other potentially confounding factors, and they found in diabetic as well as non diabetic patients. The mechanism responsible for this association has yet to be clearly described. Very few studies were carried out in India associating NAFLD with diabetic and Prediabetic patients $(6,7)$. At present India has 77.2 million people with prediabetes (8) and it is known that health complications associated with Type 2 diabetes often occur before the medical diagnosis of diabetes is made. Hence prediction of cardiovascular and renal disease in prediabetic phase itself has therapeutic as well as economic implications of our country. Hence we require an appropriate marker to identify these risks in earlier stage and a more intensive treatment to reduce the risk of future cardiovascular events and chronic kidney disease for the benefit of a larger fraternity of patients.Microalbuminuria is a known surrogate indicator of subclinical cardiovascular and renal disease as well as vascular endothelial dysfunction. The effect of NAFLD on microalbuminuria in prediabetic phase can indirectly reflect its importance as a prognostic marker for long term complications of diabetes even before the diagnosis of diabetes is made. On this background, our study aims at comparing the occurrence of microalbuminuria in patients with prediabetes group with and without NAFLD, thus, reflecting presence or absence of any additive effect of NAFLD in prediabetes in causing major vascular complications.

\section{MATERIAL AND METHODS:}

A case - control study was undertaken in the Department of General Medicine, Dr.PSIMS\&RF, Chinoutpally, Gannavaram, Krishna (Dt), Andhra Pradesh. 100 diagnosed Prediabetes with Non - alcoholic fatty liver disease (NAFLD) cases, who attended to our hospital and 100 age, sex matched prediabetes controls were included in our study group. All patients survived till the end of study period of one year duration i.e., January 2012 - december 2012. Diagnosis of Prediabetes cases was done as per the biochemical parameters FBS, HBA1c (Based on ADA criteria). Prediabetes cases were diagnosed when fasting blood glucose level of $100-125 \mathrm{mg} / \mathrm{dL}$ and Glycated hemoglobin was between 5.7 and 6.4 percent. Microalbuminuria in both cases and controls were estimated. Lipid profile, liver function tests and $\mathrm{C}$ - reactive protein were also estimated in 
both cases and controls as for supporting parameters in Prediabetes. The study was approved by the Ethics committee of our college. After fulfilling the inclusion and exclusion criteria, prior consent was obtained from the subjects.

\subsection{Inclusion criteria:}

Patients with fasting blood glucose value: $100-125 \mathrm{mg} \%$

HbA1c: $5.7-6.4 \%$ Ultra sound findings of fatty changes in liver in cases and normal echo pattern in controls Age 18 or older

\subsection{Exclusion criteria:}

Patients with alcohol, heart failure, acute febrile illness, renal, hepatic, malignant disorders, chronic illnesses, asymptomatic infections and smokers

\subsection{Sample collection and analysis:}

Both heparinised and plain blood samples were collected from each case and control. For analysis of FBS, lipid profile, liver function tests, CRP - serum was used and for HBA1c - whole blood was used. Serum glucose estimation was done by Trindler's GOD - POD method (commercial kit - ERBA - MANNHEIM), cholesterol estimation was done by CHOD - POD method (commercial kit - ERBA - MANNHEIM), Triglycerides estimation was done by GPO method (commercial kit - ERBA - MANNHEIM), HDL cholesterol estimation was done by APO protein precipitation or PTA method (ERBA - MANNHEIM), and HBA1c estimation was done by Ion exchange resin method (commercial kit - Randox Rx series). All these estimations were performed by Randox Daytona Autoanalyzer. VLDL-c or LDL-c levels of all cases and controls were calculated by using Friedwald's formula. CRP levels in all cases and controls were estimated by slide agglutination method in serial dilutions using serum. Urine samples from 24 - hour collected specimens were taken from each case and control and microalbumin estimation was done in those samples by Latex turbidimetry method (commercial kit-Euro diagnostic systems) on Randox Daytona Autoanalyzer.

\section{RESULTS:}

The microalbuminuria was present in $33 \%$ of cases and $14 \%$ of controls $(\mathrm{p}=0.0027)$, which showed microalbuminuria is more frequent in prediabetes with Non - alcoholic fatty liver disease (NAFLD) cases than prediabetes alone. Among in males, $19(38 \%)$ in cases and $9(18 \%)$ in controls were showed microalbuminuria was statistically significant $(\mathrm{p}=0.045)$. Among in females, $14(28 \%)$ in cases and $5(10 \%)$ in controls were showed microalbuminuria was statistically significant $(\mathrm{p}=0.0414)$. The mean microalbuminuria in cases was $31.64 \pm 10.53$ and in controls was $27.62 \pm 8.48(\mathrm{p}=0.0033)$ which showed microalbuminuria is more frequent in Prediabetes with Non - alcoholic fatty liver disease (NAFLD) cases, among in males mean microalbuminuria in cases was $31.33 \pm 5.51$ and in controls was $28.14 \pm 9.09(\mathrm{p}=0.0197)$, among in females mean microalbuminuria in cases was $31.27 \pm 9.41$ and in controls was $26.42 \pm 6.86(\mathrm{p}=0.023)$ were showed statistically significant mean microalbuminuria levels in males and females in cases than controls. The mean CRP in cases was $3.58 \pm 2.61$ and in controls was $2.08 \pm 0.88(\mathrm{p}=0.0001)$ which showed CRP is more significant elevation in Prediabetes with Non - alcoholic fatty liver disease (NAFLD) cases, among in males mean CRP in cases was $3.19 \pm 2.04$ and in controls was $2.12 \pm 0.95(\mathrm{p}=0.0001)$, among in females mean CRP in cases was $4.33 \pm 3.37$ and in controls was $1.99 \pm 0.74(\mathrm{p}=0.0004)$ were showed statistically significant mean microalbuminuria levels in males and females in cases than controls. The mean cholesterol in cases was $207.68 \pm 44.76$ and in controls was $190.99 \pm 24.49(\mathrm{p}=0.0013)$ which showed cholesterol is more significant elevation in Prediabetes with Non - alcoholic fatty liver disease (NAFLD) cases, The mean triglyceride level in cases was $163.89 \pm 47.83$ and in controls was $136.22 \pm 69.72(\mathrm{p}=0.0013)$ which showed mean triglyceride level is more significant elevation in Prediabetes with Non - alcoholic fatty liver disease (NAFLD) cases, The mean LDL - cholesterol level in cases was $128.79 \pm 17.27$ and in controls was $118.56 \pm 20.9(\mathrm{p}=0.0002)$ which showed mean LDL - cholesterol level is more significant elevation in Prediabetes with Non - alcoholic fatty liver disease (NAFLD) cases, The mean HDL - cholesterol level in cases was $35.43 \pm 5.33$ and in controls was $38.68 \pm 9.06(\mathrm{p}=0.0023)$ which showed mean HDL - cholesterol level is more significant elevation in Prediabetes with Non - alcoholic fatty liver disease (NAFLD) cases.

Table 1

Microalbuminuria $>30 \mathrm{mg} /$ day in cases and controls in males and females

\begin{tabular}{|l|l|l|l|l|}
\hline & Prediabetes with NAFLD & Prediabetes & Chi - square value & P value \\
\hline Total & $\mathbf{3 3}$ & $\mathbf{1 4}$ & $\mathbf{9 . 0 1 1}$ & $\mathbf{0 . 0 0 2 7}$ \\
\hline Males & $\mathbf{1 9}$ & $\mathbf{9}$ & 4.018 & $\mathbf{0 . 0 4 5}$ \\
\hline females & $\mathbf{1 4}$ & $\mathbf{5}$ & 4.159 & $\mathbf{0 . 0 4 1 4}$ \\
\hline
\end{tabular}


Table 2

Mean Microalbuminuria $>30 \mathrm{mg} /$ day in cases and controls in males and females

\begin{tabular}{|l|l|l|l|}
\hline & $\begin{array}{l}\text { Prediabetes with } \\
\text { NAFLD }\end{array}$ & Prediabetes & P value \\
\hline Total & $31.64 \pm 10.53$ & $27.62 \pm 8.48$ & $\mathbf{0 . 0 0 3 3}$ \\
\hline Males & $31.33 \pm 5.51$ & $28.14 \pm 9.09$ & $\mathbf{0 . 0 1 9 7}$ \\
\hline females & $31.27 \pm 9.41$ & $26.42 \pm 6.86$ & $\mathbf{0 . 0 2 3}$ \\
\hline
\end{tabular}

Table 3

Mean CRP levels in cases and controls in males and females

\begin{tabular}{|l|l|l|l|}
\hline & $\begin{array}{l}\text { Prediabetes with } \\
\text { NAFLD }\end{array}$ & Prediabetes & P value \\
\hline Total & $3.58 \pm 2.61$ & $2.08 \pm 0.88$ & 0.0001 \\
\hline Males & $3.19 \pm 2.04$ & $2,12 \pm 0.95$ & 0.0001 \\
\hline females & $4.33 \pm 3.37$ & $1.99 \pm 0.74$ & 0.0004 \\
\hline
\end{tabular}

Table 4

Mean lipid levels in cases and controls in males and females

\begin{tabular}{|l|l|l|l|}
\hline & NAFLD with Prediabetes & $\begin{array}{l}\text { Prediabetes without } \\
\text { NAFLD }\end{array}$ & P value \\
\hline Cholesterol & $207.68 \pm$ & $190.99 \pm$ & 24.49 \\
& 44.76 & $136.22 \pm$ & 0.0013 \\
\hline Triglycerides & $163.89 \pm$ & 69.72 & 0.0013 \\
& 47.83 & $118.56 \pm$ & \\
\hline LDL & $128.79 \pm$ & 20.9 & 0.0002 \\
& 17.27 & $38.68 \pm$ & \\
\hline HDL & $35.43 \pm$ & 9.06 & 0.0023 \\
& 5.33 & & \\
\hline
\end{tabular}

Table 5

Mean HbA1c levels in cases and controls in males and females

\begin{tabular}{|l|l|l|l|}
\hline & $\begin{array}{l}\text { NAFLD with } \\
\text { Prediabetes }\end{array}$ & $\begin{array}{l}\text { Prediabetes without } \\
\text { NAFLD }\end{array}$ & P value \\
\hline \multirow{2}{*}{ Mean HbA1c } & $\begin{array}{l}5.970 \pm \\
0.179\end{array}$ & $\begin{array}{l}5.895 \pm \\
0.125\end{array}$ & \multirow{2}{*}{0.0007} \\
\hline Males & $5.968 \pm$ & $5.899 \pm$ & \\
& 0.178 & 0.125 & 0.0089 \\
\hline females & $5.974 \pm$ & $5.887 \pm$ & 0.0337 \\
& 0.183 & 0.128 & \\
\hline
\end{tabular}

\section{Discussion:}

Non - alcoholic fatty liver disease (NAFLD) represents a spectrum of disorders characterized by predominantly macro - vesicular hepatic steatosis, that occur in individuals even in the absence of consumption of alcohol in amounts considered harmful to the liver (8). Most individuals who are suffering from NAFLD are asymptomatic (9). However, a subset of individuals progresses to more severe manifestations including non alcoholic steatohepatitis (NASH), fibrosis, cirrhosis and liver failure $(10,11)$. The pathogenesis of the NAFLD is yet to be completely understood. It involves deposition of excess fat in the liver; followed by some patients by increased fatty acid oxidation, oxidative stress and cytokine production, resulting in progression to steatohepatitis and fibrosis. Various pathogenic mechanisms that play a role include cytokines (tumor necrosis factor (TNF) - a, adiponectin, resistin, leptin, interleukins, transforming growth factor etc.) (12-14) Epidemiological studies show a prevalence of NAFLD in around 9\% to 32\% of general population in India with higher prevalence in those with overweight or obesity and those with diabetes or prediabetes. In our study we studied effect of NAFLD on microalbuminuria in Prediabetic phase, as it is an early and sensitive marker of future cardio vascular events even in healthy subjects. Microalbuminuria seems to reflect a state of pathophysiologic vascular dysfunction that makes an individual susceptible to organ damage. High levels of 
albuminuria may already be found in young children and reflect a normal physiologic variation in endothelial function associated with cardiovascular and renal risk at later age. Intervention strategies aimed at repairing this vascular could be very useful not only in secondary prevention but also in primary prevention. (15) There was an additive effect of NAFLD and prediabetes on microalbuminuria and thereby on cardiovascular and renal risk, the basis for which is not clearly understood. Our results didn't show significant difference in factors like blood pressure, obesity, mean age and sex between the two study groups of prediabetics and this confirms the effect of NAFLD on microalbuminuria independent of above said factors. Proinflammatory cytokines from injured hepatocytes, responsible for local inflammation may also be responsible for sub clinical systemic inflammatory response reflected by increased microalbuminuria in NAFLD group, also atherogenic dyslipidemia may contribute to the endothelial dysfunction found in the NAFLD group. Finally, NAFLD may worsen whole-body insulin resistance and hyperglycemia $(16,17)$, which may contribute to disease progression and initiation of chronic kidney disease and coronary artery disease. This is supported by the observation, that the HbAlc was higher in the study group with NAFLD than in hose without NAFLD.The potential implication of our findings for patient care is that in people with prediabetes the causal detection of NAFLD during ultrasound examination should alert clinicians for higher chances of disease progression as well as increased susceptibility for other chronic diabetic complications (including CKD and CAD) as much as the risk for advancing liver disease. Thus identifying people with NAFLD with prediabetes would highlight a subgroup of individuals who should be targeted for more intensive therapy and increased surveillance to decrease the risk for developing diabetes, as well as CKD and CAD events. Because liver biopsies were not available in this study, we can't exclude the possibility of a differential relationship between histologic severity of NAFLD and CKD and CAD risk. It is also not known whether improving NAFLD will ultimately prevent the progression of disease and prevention of macrovascular complications. Hence there is clear need for further prospective studies before using NAFLD as a marker for risk of long term complications in Prediabetic patients.

\section{CONCLUSIONS}

Microalbuminuria levels were found to be significantly higher in 33\% of Prediabetes patients with NAFLD when compared to those without. High mean microalbuminuria, mean CRP levels with significant elevated lipid levels in Prediabetes patients with NAFLD than without NAFLD. Larger studies are required to clarify the significance of development of complications and consider the microalbuminuria as a regular and routine screening marker to avoid sub clinical target organ damage.

\section{BIBLIOGRAPHY:}

[1] Angulo P. Nonalcoholic fatty liver disease. N Engl J Med 2002; 346: 1221-1231.

[2] Targher G, Arcaro G. Non-alcoholic fatty liver disease and increased risk of cardiovascular disease. Atherosclerosis 2007; 191:2:235-240.

[3] Marchesini G, Bugianesi E, Forlani G, Cerrelli F, Lenzi M, Manini R, et al. Nonalcoholic fatty liver, steatohepatitis, and the metabolic syndrome. Hepatology 2003;4: 917-923

[4] TARGHER G., BERTOLINI L., POLI F., RODELLA S., SCALA L. et al., Nonalcoholic fatty liver disease and risk of future cardiovascular events among type 2 diabetic patients. Diabetes, 2005, 54:3541-3546

[5] TARGHER G., BERTOLINI L., RODELLA S. et al., Nonalcoholic Fatty Liver Disease Is Independently Associated With an Increased Incidence of Cardiovascular Events in Type 2 Diabetic Patients. Diabetes Care, 2007, 30:2119-2121.

[6] AK Agarwal*, Vineet Jain* et al. Prevalence of Non-Alcoholic Fatty Liver Disease and its Correlation with Coronary Risk Factors in Patients with Type 2 Diabetes JAPI • JUNE 2011 • VOL. 59

[7] Viswanathan, Mahesh Kadiri et.al.association of non-alcoholic fatty liver disease with diabetic microvascular and macrovascular complications in South Indian diabetic subjects. International Journal of Diabetes in Developing Countries | October-December 2010 | Volume 30 | Issue 4 diabetolgia 2011 Dec;54(12):3022-7. doi: 10.1007/s00125-011-2291-5. Epub 2011

[8] Marchesini G, Moscatiello S, Agostini F, Villanova N, Festi D. Treatment of non-alcoholic fatty liver disease with focus on emerging drugs. Expert Opin Emerg Drugs. 2011; 1: 121-136. doi:10.1517/14728214.2011.531700

[9] Tarquini R, Lazzeri C, Boddi M, Marra F, Abbate R, Gensini GF. [Non-alcoholic fatty liver disease: a new challenge for cardiologists]. G Ital Cardiol

[10] Clark J, Brancati F, Diehl A. The prevalence and etiology of elevated aminotransferase levels in the United States. Am J Gastroenterol. 2003; 98: 960-967. doi:10.1111/j.1572-0241.2003.07486.x

[11] Jin H, Gu Z, Yu C, Li Y. Association of non-alcoholic fatty liver disease with type 2 diabetes: clinical features and independent risk factors in diabetic fatty liver patients. Hepatobiliary Pancreat Dis Int. 2005; 4: 389-392. PMid:16109522

[12] Angulo P. Nonalcoholic fatty liver disease. N Engl J Med. 2002;346:1221-31.

[13] AGA technical review on nonalcoholic fatty liver disease. Gastroenterology. 2002;123:1705-25.

[14] Neuschwander-Tetri BA, Caldwell SH. Nonalcoholic steatohepatitis: summary of an AASLD single topic conference. Hepatology. 2003;37:1202-19.

[15] Dick de Zeeuw*, Hans-Henrik Parving † et .al . Microalbuminuria as an Early Marker for Cardiovascular Disease. J Am Soc Nephrol 17: 2100-2105, 2006. doi: 10.1681/ASN.2006050517

[16] Adams LA, Angulo P, Lindor KD: Nonalcoholic fatty liver disease. CMAJ172 : 899 -905, 2005

[17] Targher G: Non-alcoholic fatty liver disease, the metabolic syndrome and the risk of cardiovascular disease: The plot thickens. Diabet Med24 : $1-6,2007$ 